\title{
Water nucleation at extreme supersaturation
}

\section{Journal Article}

Author(s):

Lippe, Martina; Chakrabarty, Satrajit; Chakrabarty, Satrajit; Tanaka, Kyoko K.; Signorell, Ruth (D)

Publication date:

2018-12-28

Permanent link:

https://doi.org/10.3929/ethz-b-000314682

\section{Rights / license:}

In Copyright - Non-Commercial Use Permitted

Originally published in:

The Journal of Chemical Physics 149(24), https://doi.org/10.1063/1.5052482

\section{Funding acknowledgement:}

172472 - Phase Transitions of Ultrafine Aerosol Particles: Condensation, Freezing, and Metal Formation in Confined Systems (SNF) 


\section{Water nucleation at extreme supersaturation}

Cite as: J. Chem. Phys. 149, 244303 (2018); https://doi.org/10.1063/1.5052482

Submitted: 18 August 2018 . Accepted: 21 November 2018 . Published Online: 26 December 2018

Martina Lippe, Satrajit Chakrabarty, Jorge J. Ferreiro, Kyoko K. Tanaka, and Ruth Signorell (D)

\section{COLLECTIONS}

Paper published as part of the special topic on JCP Editors' Choice 2018

F This paper was selected as Featured
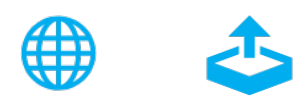

\section{ARTICLES YOU MAY BE INTERESTED IN}

First laboratory results of supersaturated water nucleation characteristics at low temperatures

Scilight 2018, 520005 (2018); https://doi.org/10.1063/1.5085733

Overview: Homogeneous nucleation from the vapor phase-The experimental science The Journal of Chemical Physics 145, 211702 (2016); https://doi.org/10.1063/1.4962283

Collective hydrogen-bond rearrangement dynamics in liquid water

The Journal of Chemical Physics 149, 244504 (2018); https://doi.org/10.1063/1.5054267

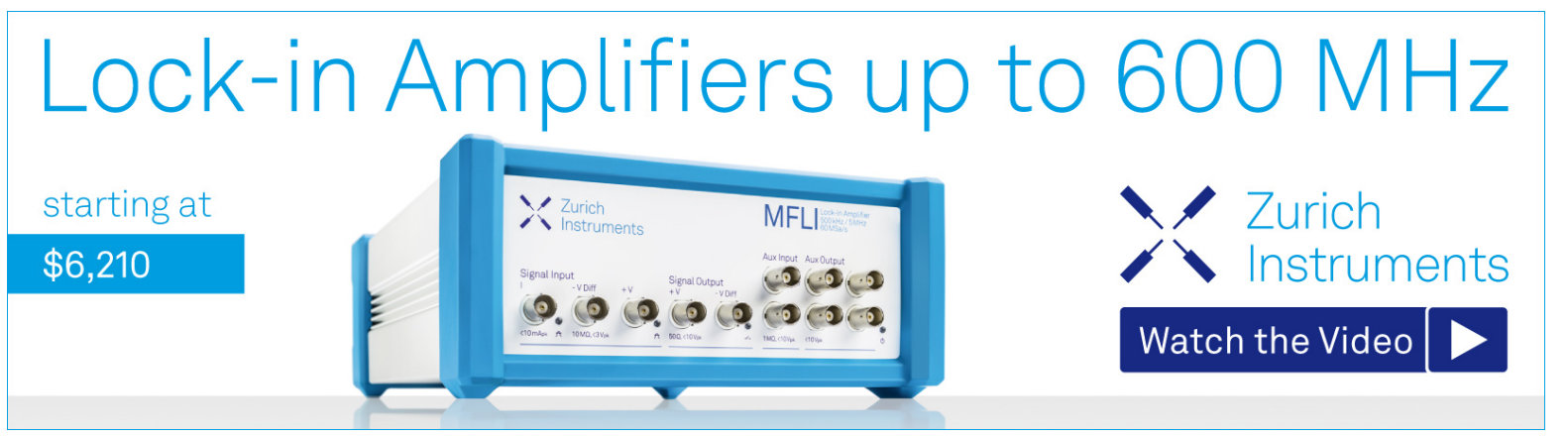




\title{
Water nucleation at extreme supersaturation
}

\author{
Martina Lippe, ${ }^{1}$ Satrajit Chakrabarty, ${ }^{1}$ Jorge J. Ferreiro, ${ }^{1}$ Kyoko K. Tanaka, ${ }^{2}$ \\ and Ruth Signorell ${ }^{1}$ \\ ${ }^{1}$ Laboratory of Physical Chemistry, ETH Zürich, Vladimir-Prelog Weg 2, CH-8093 Zürich, Switzerland \\ ${ }^{2}$ Institute of Low Temperature Science, Hokkaido University, Sapporo 060-0819, Japan
}

(Received 18 August 2018; accepted 21 November 2018; published online 26 December 2018)

\begin{abstract}
We report water cluster formation in the uniform postnozzle flow of a Laval nozzle at low temperatures of 87.0 and $47.5 \mathrm{~K}$ and high supersaturations of $\ln S \sim 41$ and 104, respectively. Cluster size distributions were measured after soft single-photon ionization at $13.8 \mathrm{eV}$ with mass spectrometry. Critical cluster sizes were determined from cluster size distributions recorded as a function of increasing supersaturation, resulting in critical sizes of 6-15 and 1, respectively. Comparison with previous data for propane and toluene reveals a systematic trend in the nucleation behavior, i.e., a change from a steplike increase to a gradual increase of the maximum cluster size with increasing supersaturation. Experimental nucleation rates of $5 \cdot 10^{15} \mathrm{~cm}^{-3} \mathrm{~s}^{-1}$ and $2 \cdot 10^{15} \mathrm{~cm}^{-3} \mathrm{~s}^{-1}$ for $\ln S \sim 41$ and 104 , respectively, were retrieved from cluster size distributions recorded as a function of nucleation time. These lie 2-3 orders of magnitude below the gas kinetic collision limit assuming unit sticking probability, but they agree very well with a recent prediction by a master equation model based on ab initio transition state theory. The experimental observations are consistent with barrierless growth at $47.5 \mathrm{~K}$, but they hint at a more complex nucleation behavior for the measurement at 87.0 K. Published by AIP Publishing. https://doi.org/10.1063/1.5052482
\end{abstract}

\section{INTRODUCTION}

Since water coexists in all three phases (gaseous, liquid, and solid) on earth, phase transitions play an important role not only in cloud formation in earth's atmosphere but also in various biological and technical processes. However, the first step of a phase transition-referred to as nucleationis still poorly understood and generally hard to observe and quantify. This also holds for homogeneous nucleation from the gas phase, which is the subject of the present work. Water nucleation has been extensively studied over an extended range of temperatures and supersaturations of $T \sim 200-400 \mathrm{~K}$ and $S \sim 3-150$, respectively. For an in-depth review of water nucleation experiments, we refer the reader to Refs. 1-3 and references therein. Here, we only briefly mention previous vapor phase nucleation studies of water which, similar to our study, were performed in Laval nozzles at cryogenic temperatures. Moses and Stein ${ }^{4}$ and later Wyslouzil and co-workers ${ }^{2,5-8}$ investigated water nucleation using a combination of pressure trace measurements, light scattering techniques, and X-ray and small angle neutron scattering, from which they extracted homogeneous nucleation rates. Recently, Bourgelais et al. ${ }^{9}$ observed water cluster formation in Laval nozzles at low temperatures $(T \sim 23-69 \mathrm{~K})$ with mass spectrometry after electron ionization. From these data, the authors retrieved information on the early growth kinetics of small water clusters.

The present work reports the first laboratory measurements of water nucleation to provide direct molecular level information such as critical cluster sizes at temperatures below $87 \mathrm{~K}$. This is achieved by monitoring cluster size distributions during nucleation in the uniform postnozzle flow of a Laval expansion ${ }^{10-13}$ with mass spectrometry after single-photon vacuum ultraviolet (VUV) ionization. ${ }^{14-17}$ It has recently been shown that in contrast to electron ionization, singlephoton vacuum ultraviolet ionization close to the threshold is a soft ionization method even for weakly bound molecular clusters. ${ }^{14,15,18,19}$

Water nucleation has also been studied with computer simulations. ${ }^{20-25}$ Probably the most advanced simulations were reported by Angélil et al. ${ }^{24}$ who performed large scale molecular dynamics (MD) simulations using up to $\sim 4 \cdot 10^{6}$ particles covering a supersaturation range of $S \sim 3-24$. This allowed the retrieval of nucleation rates down to $\sim 10^{19} \mathrm{~cm}^{-3} \mathrm{~s}^{-1}$, i.e., very close to the highest experimentally measured rates of $\sim 10^{17} \mathrm{~cm}^{-3} \mathrm{~s}^{-1}, 5,6,26$ A true overlap between experiment and simulations was recently achieved by the same research team for computationally much less demanding Lennard-Jones systems. ${ }^{27-29}$

The thermodynamic requirement to induce vapor phase nucleation in an experiment is often expressed by the approximate condition that the vapor supersaturation $S=p_{\text {cond }} / p_{\text {eq }}(T)$ must exceed 1 , where $p_{\text {cond }}$ is the partial pressure of the condensable and $p_{\mathrm{eq}}(T)$ is the equilibrium vapor pressure of the condensable at temperature $T$. Kinetically, the formation of a critical nucleus of size $n_{\mathrm{c}}$ (number of molecules per cluster) is considered to be the rate-determining step. $n_{\mathrm{c}}$ corresponds to the cluster size where the growth rate matches the decay (evaporation) rate. This is typically associated with a maximum in the $n$-dependent Gibbs free energy of cluster formation $\Delta G\left(n_{\mathrm{c}}\right)$. An increase in $S$ results in a simultaneous decrease of $n_{\mathrm{c}}$ and $\Delta G\left(n_{\mathrm{c}}\right)$. However, nucleation can only be observed 
when it takes place in the time window that is accessible by a certain experimental setup. To account for this, we choose to refer to experimental conditions where $S>1$ but nucleation is not yet observable as subcritical conditions, while supercritical conditions refer to conditions for which nucleation has already taken place in the experiment. ${ }^{11-13}$

Nucleation is very sensitive to changes in the actual conditions $(p, T$, and $S)$. Our nucleation studies are performed in the uniform postnozzle flow of a Laval expansion which ensures working under constant and well-known experimental conditions. $S$ can be systematically tuned by varying $p_{\text {cond }}$ and $T$ in the postnozzle flow in fine steps. In our experiments, we usually work at very high values of $S$, typically exceeding $10^{12} .^{12}$ At such high supersaturations, it is no longer clear whether nucleation takes place in the presence or in the absence of a barrier (spinodal decomposition). ${ }^{30-32}$ This is also an interesting regime for modeling approaches because it allows stringent model tests and promises closing the gap between experiments and molecular dynamics simulations. ${ }^{24,28}$ We can probe the uniform postnozzle flow at any position along the flow with a time-resolution of $\sim 2 \mu \mathrm{s} .{ }^{12}$ The length of the uniform postnozzle flow and the velocity of the molecular beam determine the time-window of up to a few hundred $\mu$ s that is accessible by our experiment. As mentioned in our previous work, ${ }^{13}$ the long-term goal of our experiments is to provide molecular level data on nucleation for different substances. These data in combination with modeling approaches ${ }^{9,27,30,33-37}$ can provide deeper insight into gas phase nucleation at the molecular level.

\section{EXPERIMENT}

\section{A. Instrumentation}

Figure 1 shows a simplified scheme of the experimental setup, which has been described in detail in previous publications. ${ }^{10-13}$ Two feeding valves (not shown) with opening times of $6 \mathrm{~ms}$ supplied the gas mixture (water vapor, carrier gas, and internal standard) to the stagnation volume of the Laval nozzle at a stagnation pressure and temperature of $p_{0}$ and $T_{0}$, respectively. Mass flow controllers were used to regulate the flows of the carrier gases (argon, PanGas 5.0 or nitrogen, PanGas 5.0) and the internal standard

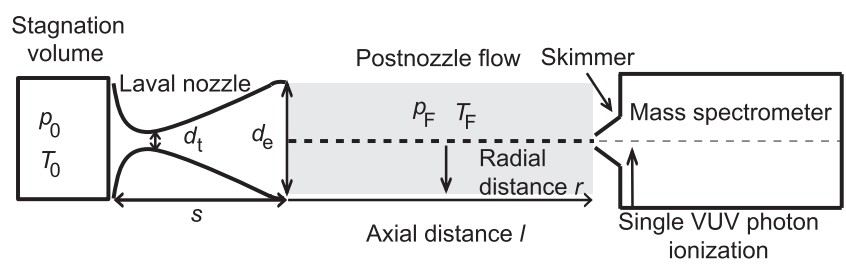

FIG. 1. Schematic of the experimental setup. ${ }^{12,13} p_{0}$ is the stagnation pressure, $T_{0}$ is the stagnation temperature, $p_{\mathrm{F}}$ is the flow pressure, and $T_{\mathrm{F}}$ is the flow temperature. $d_{\mathrm{t}}, d_{\mathrm{e}}$, and $s$ are the throat diameter, the exit diameter, and the length of the Laval nozzle, respectively. $r$ is the radial distance from the center of the expansion. The axial distance $l$ is the distance between the nozzle exit and the skimmer. $l$ can be varied, which corresponds to a variation in the nucleation time $t$. The central part of the expansion is sampled into the mass spectrometer by a skimmer, where the clusters are ionized and detected. (methane gas, Messer Schweiz 5.5). A vapor mixture of a well-defined amount of water and a carrier gas was generated in the evaporation unit after the liquid flow meter (LFM) (Bronkhorst $\mu$-Flow, accuracy: $\pm 0.5 \%$ RD plus $\pm 0.1 \%$ FS). To prevent water condensation, the delivery tubes that connect the LFM with the Laval nozzle stagnation volume were heated. The uniform flow at the exit of the Laval nozzle was extended into the postnozzle region by matching the flow pressure $p_{\mathrm{F}}$ to the background pressure in the chamber. The maximum length of the uniform postnozzle flow is typically $l_{\max }=100-150 \mathrm{~mm}$. The Laval nozzle is mounted on a translation stage which allowed us to change the axial distance $l$ between the exit of the Laval nozzle and the skimmer entrance in steps as small as $1 \mathrm{~mm}$ (Fig. 1). A change in the axial distance corresponds to a change in time. The accessible time scale of our experiment is on the order of a few hundred $\mu \mathrm{s}$ with a time-resolution of $\sim 2 \mu \mathrm{s}$, which is limited by the spot size of the ionizing laser $(\sim 1 \mathrm{~mm}$, see below). As described in Refs. 10, 11, and 38, the experimental Mach numbers $M$ at distances $l$ were determined from the measured stagnation and impact pressures $p_{0}$ and $p_{\mathrm{I}}$, respectively, using the RayleighPitot relation. The flow pressure $p_{\mathrm{F}}$ was measured directly using a Baratron capacitance manometer and the flow temperature $T_{\mathrm{F}}$ was calculated from energy conservation assuming isentropic relations and ideal gas behavior (Fig. 1). We consider the uncertainty introduced by the latter two assumptions as insignificant compared with the quoted uncertainty of the impact pressure measurements. To verify the uniformity of the postnozzle flow, $p_{\text {I }}$ was measured not only as a function of the axial $(l)$ distance but also as a function of the radial $(r)$ distances. Table I summarizes the characteristics of the Laval nozzles that were used in this study. $T_{\mathrm{F}}$ is the flow temperature at an axial position of $l=60 \mathrm{~mm}$ where the nucleation measurements at varying supersaturations (Sec. III A) were performed. $\bar{M}$ and $\overline{T_{\mathrm{F}}}$ correspond to the axially averaged Mach numbers and flow temperatures, respectively, with standard deviations

TABLE I. Physical dimensions and flow properties of the Laval nozzles used in this study. $s, d_{\mathrm{t}}$, and $d_{\mathrm{e}}$ are the lengths, the throat diameters, and the exit diameters of the nozzles, respectively. $p_{\mathrm{F}}$ is the flow pressure for the optimal postnozzle flow conditions and $p_{\text {cond }}$ is the partial pressure of the condensable in the postnozzle flow. $l_{\max }$ is the maximal length of the uniform postnozzle flow. $\bar{M}$ and $\overline{T_{\mathrm{F}}}$ are the axially averaged Mach number and flow temperature, respectively. $T_{\mathrm{F}}$ is the flow temperature at the axial distance $l=60 \mathrm{~mm}$ where the nucleation measurements for varying $S$ were performed (Sec. III A). Note that the labels Mach 4.0 and Mach 6.0 refer to a specific nozzle and do not necessarily match the experimental Mach number $\bar{M}$.

\begin{tabular}{lccc}
\hline \hline & $\begin{array}{c}\text { Mach 4.0 } \\
\mathrm{H}_{2} \mathrm{O} \text { in Ar }\end{array}$ & $\begin{array}{c}\text { Mach 4.0 } \\
\mathrm{H}_{2} \mathrm{O} \text { in } \mathrm{N}_{2}\end{array}$ & $\begin{array}{c}\text { Mach 6.0 } \\
\mathrm{C}_{3} \mathrm{H}_{8} \text { in Ar }\end{array}$ \\
\hline$s(\mathrm{~mm})$ & 105.0 & 105.0 & 101.0 \\
$d_{t}(\mathrm{~mm})$ & 8.1 & 8.1 & 4.1 \\
$d_{e}(\mathrm{~mm})$ & 27.1 & 27.1 & 23.4 \\
$p_{\mathrm{F}}(\mathrm{Pa})$ & 40 & 42 & 35 \\
$p_{\text {cond }}(\mathrm{Pa})$ & 0.067 & 0.321 & 0.019 \\
$l_{\text {max }}(\mathrm{mm})$ & 100 & 100 & 100 \\
$\bar{M}$ & $3.98 \pm 0.06$ & $2.99 \pm 0.05$ & $5.21 \pm 0.11$ \\
$\overline{T_{F}}(\mathrm{~K})$ & $47.5 \pm 1.2$ & $87.0 \pm 1.3$ & $29.4 \pm 1.2$ \\
$T_{F}(\mathrm{~K})$ & $48.5 \pm 0.3$ & $85.6 \pm 0.3$ & $31.0 \pm 1.3$ \\
\hline \hline
\end{tabular}


$(1 \sigma)$ determined from 40 individual measurements along the flow axis.

The central part of the expansion was sampled into the mass spectrometer (Fig. 1) by a skimmer (1 $\mathrm{mm}$ in diameter), where the neutral clusters were ionized by single-photon ionization with a home-built table top vacuum ultraviolet (VUV) laser. ${ }^{39} \mathrm{~A}$ fast intracluster proton transfer takes place after single-photon ionization resulting in protonated water species in the mass spectrum. ${ }^{15,17,40-42}$ The VUV-light was generated by resonance enhanced two-color four-wave mixing in a krypton expansion at $20 \mathrm{~Hz} \cdot{ }^{10,39}$ We used a fixed photon energy of $13.8 \mathrm{eV}(89.8 \mathrm{~nm})$ close to the lowest ionization threshold of water clusters at a photon flux in the range of $\sim 10^{11}-10^{12}$ photons $/\left(\mathrm{cm}^{2}\right.$ pulse $)$. As explained in Refs. $14-17$, this ensures soft ionization even for weakly bound molecular clusters. The cluster ions were extracted by a Wiley McLaren type extractor with voltages of up to $30 \mathrm{kV}$ and detected by a multichannel plate (MCP) detector. We used high extraction voltages to optimize the sensitive detection. The monomer ion signal was $\sim 10^{3}-10^{4}$ higher than the cluster ion signals so that the monomer and the clusters had to be recorded in separate measurements using different experimental settings. ${ }^{12}$ Furthermore, to avoid saturation effects caused by the monomer in the cluster measurements, the monomer ion was selectively deflected by a pulsed plate located in front of the MCP detector for the cluster measurements. ${ }^{10,11,13}$

\section{B. Cluster number concentrations from mass spectra}

The determination of nucleation rates from the experiments in which the nucleation time $t$ was varied (Sec. III B) required the knowledge of the absolute number concentration $N_{\mathrm{n}}^{\mathrm{H}_{2} \mathrm{O}}$ of clusters with $n$ molecules in each of the size distributions recorded at different times $t$. This information was extracted from the experimental cluster spectra using a known amount of $\mathrm{CH}_{4}$ gas that was added to the gas mixture as an internal standard. Note that water monomers in general cannot be used as a concentration standard because of varying trace amounts of water from the background of the mass spectrometer. There is also an issue with potential monomer depletion due to cluster growth. For every measurement, the same amount of methane (number concentration $N^{\mathrm{CH}_{4}}$ ) was used, which corresponded to approximately $1 \%$ of the sample gas mixture. $\mathrm{CH}_{4}$ fulfills all requirements for a useful internal standard: (i) It does not influence the uniformity of the Laval expansion. (ii) It does not cluster with itself and it does not co-cluster with the condensable under investigation. (iii) It does not undergo any reactions with the condensable. (iv) $\mathrm{The} \mathrm{CH}_{4}$ ion mass does not overlap with the masses of the clusters in the mass spectrum. (v) The photoionization cross section $\sigma_{\mathrm{VUV}, \mathrm{CH}_{4}}$ at $13.8 \mathrm{eV}$ is known. ${ }^{43} N_{\mathrm{n}}^{\mathrm{H}_{2} \mathrm{O}}$ was then determined from the ion signal $I_{\mathrm{n}}$ of the water cluster with $n$ molecules, the ion signal $I_{\mathrm{CH}_{4}}$ of the $\mathrm{CH}_{4}$ monomer, the number concentration $N^{\mathrm{CH}_{4}}$ of the $\mathrm{CH}_{4}$ monomer, and the photoionization cross section of the $\left(\mathrm{H}_{2} \mathrm{O}\right)_{n}$ cluster and of the $\mathrm{CH}_{4}$ monomer $n \sigma_{\mathrm{H}_{2} \mathrm{O}}$ and $\sigma_{\mathrm{CH}_{4}}$, respectively, ${ }^{43,44}$

$$
N_{\mathrm{n}}^{\mathrm{H}_{2} \mathrm{O}}=\frac{I_{\mathrm{n}}}{I_{\mathrm{CH}_{4}}} \frac{\sigma_{\mathrm{CH}_{4}}}{\sigma_{\mathrm{H}_{2} \mathrm{O}} \cdot n} \cdot N^{\mathrm{CH}_{4}} .
$$

Consistent with physical considerations, we have assumed that for single-photon VUV ionization resulting in singly charged cluster ions, the photoionization cross section of a cluster with $n$ molecules is $n$ times the photoionization cross section of the water monomer $\sigma_{\mathrm{H}_{2} \mathrm{O}} \cdot{ }^{44}$ Equation (1) is valid only if the detection efficiency (product of ion transmission ${ }^{45}$ and detection efficiency at the MCP) of ions in the linear time-of-flight mass spectrometer is independent of $n$. The detection efficiency primarily depends on the ion velocity with a typical threshold behavior. Typical mass spectra recorded under nucleation conditions consist of small clusters with $n<100 ;{ }^{12,13}$ in the case of water, this corresponds to $\mathrm{m} / \mathrm{z}$ values below 2000 . Our ions are accelerated with extraction voltages close to $30 \mathrm{kV}$, which results in an impact velocity of $1 \cdot 10^{5} \mathrm{~ms}^{-1}$ for a cluster mass of 540. Figure 2(a) shows water cluster mass
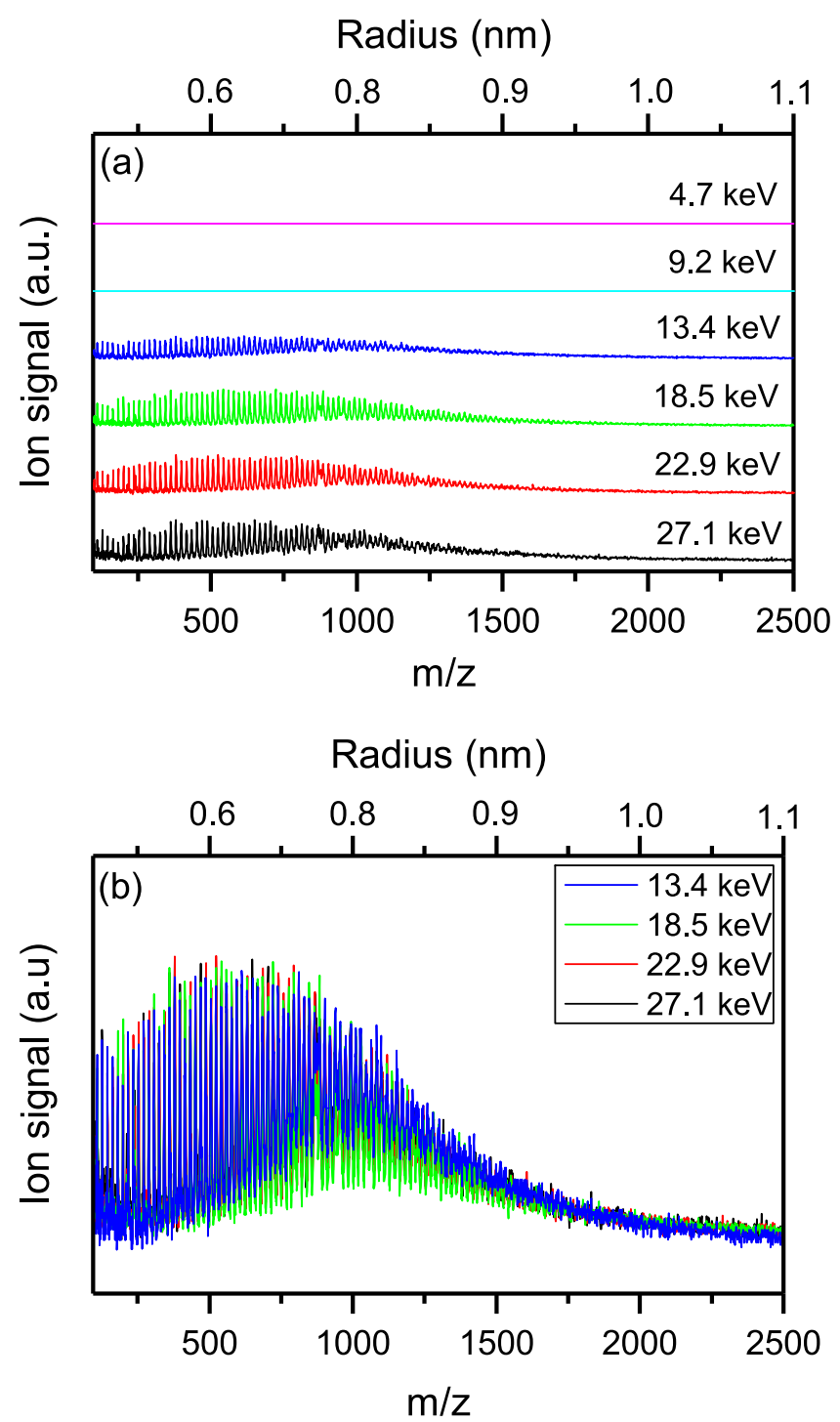

FIG. 2. (a) Comparison of mass spectra recorded at different ion extraction voltages between 4.7 and $27.1 \mathrm{keV}$ corresponding to different impact velocities of the ions which impinge on the MCP detector. ${ }^{67}$ While no ion signals can be detected at low extraction voltages, the absolute ion signal does no longer vary above $\sim 18 \mathrm{keV}$. (b) Mass spectra for voltages above $\sim 13 \mathrm{keV}$ normalized to the same absolute signal intensities. In this range, the detection efficiency is independent of $n$. The water clusters were generated in the Mach 4 nozzle using Ar as a carrier gas. 
spectra recorded at different extraction voltages between 4.7 and $27.1 \mathrm{keV}$, and Fig. 2(b) shows the same spectra for voltages above $13.4 \mathrm{keV}$ after normalizing the spectra to the same absolute ion signal. The fact that the normalized spectra recorded at the higher extraction voltages are identical proves that the detection efficiency no longer depends on the ion velocity and hence is independent of $n$. This is not the case for lower extraction voltages, when the shape of the distribution still changes with increasing extraction voltage [Fig. 2(a)]. At sufficiently high impact velocities, by contrast, the detection efficiency becomes not only independent of $n$ but also appears to reach a plateau as a function of the extraction voltage. Figure 2(a) also illustrates that high extraction voltages close to $30 \mathrm{kV}$ result in a significant gain in the absolute ion intensity and that at impact energies of less than $\sim 9 \mathrm{keV}$ no ion signals can be detected. Especially for mass spectra recorded under nucleation conditions, high extraction voltages are thus crucial for the sensitive detection of the clusters. Finally, we point out that monomer depletion and heat release during nucleation is negligible in our experiments, which is important to ensure constant experimental conditions, e.g., constant $S$. Many different factors contribute to the uncertainty in the experimental nucleation rate constants (Sec. III B), such as the approximations used in the determination of the flow temperature and the uncertainties in the determination of the cluster number concentrations. Some of the uncertainties are rather difficult to quantify. A very rough estimate suggests an overall uncertainty in the nucleation rate constants of around one order of magnitude.

\section{NUCLEATION RESULTS AND DISCUSSION}

Our experimental setup allows us to perform two different types of nucleation experiments. The first type of experiment is described in detail in our previous studies. ${ }^{11-13}$ In these experiments, we record cluster size distributions at a fixed axial distance $l$ in the postnozzle flow (corresponding to a fixed time $t$ ) while systematically increasing the supersaturation $S$ in very fine steps across the region where nucleation takes place, i.e., from subcritical to supercritical conditions (Sec. III A). These studies provide a direct experimental measurement of the critical cluster size $n_{\mathrm{c}}$. In the second type of experiment, the nucleation time $t$ is systematically increased by increasing the distance $l$ at constant $S$ (Sec. III B). Direct experimental access to the nucleation rate $J$ is obtained through $t$ and $N_{\mathrm{n}}^{\mathrm{H}_{2} \mathrm{O}}$.

\section{A. Critical cluster sizes from varying the supersaturation}

Figure 3 shows mass spectra of $\mathrm{H}_{2} \mathrm{O}$ clusters recorded for increasing $S$ at a fixed axial distance $l=60 \mathrm{~mm}$ and constant flow temperature $T_{\mathrm{F}} . S$ was systematically increased by increasing the water partial pressure $p_{\text {cond }}$ in the gas mixture of the postnozzle flow. The black line follows the maximal cluster size $n_{\max }$ recorded in each mass spectrum. At low $p_{\text {cond }}$, the cluster intensities are very small, starting to increase at concentrations around $0.11 \%$ (concentrations are specified throughout in mole percent). This corresponds to a gradual

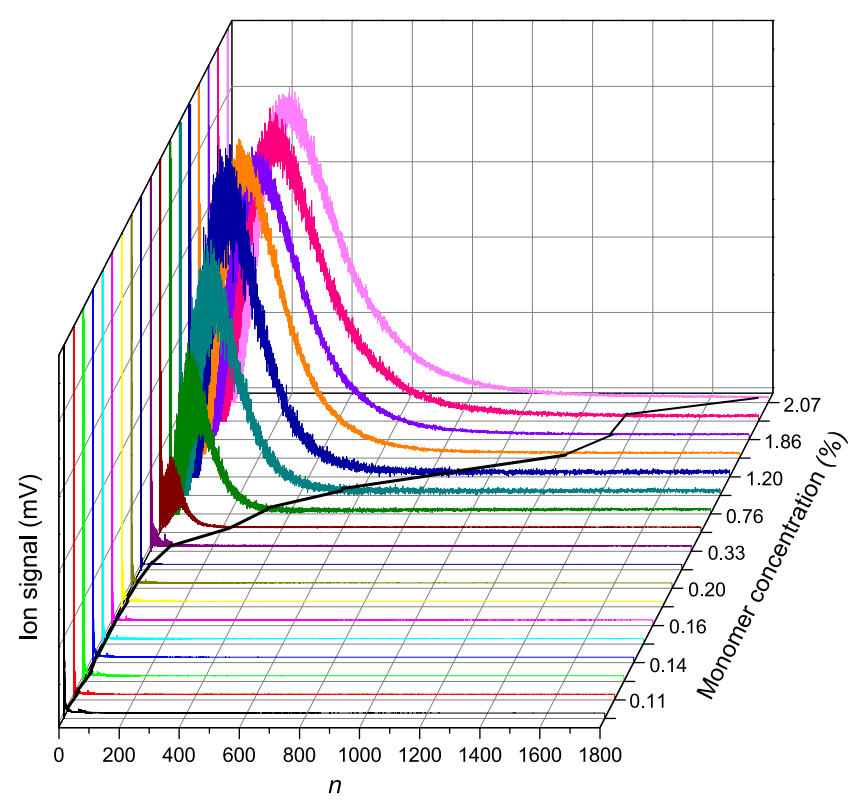

FIG. 3. Mass spectra of water clusters generated in the Mach 4 nozzle using $\mathrm{Ar}$ as the carrier gas as a function of the water monomer concentration (given in terms of mole percent). $n$ is the number of water molecules per cluster. The black line visualizes the course of the maximum cluster size $n_{\max }$ with increasing monomer concentration. It indicates the extent of cluster growth. This is an example for a gradual increase of the maximum cluster size, which hints at a barrierless process.

increase of the cluster size with increasing $S$ (see below) as the conditions change from subcritical to supercritical. By contrast, a steplike increase of the cluster size with increasing $S$ (see below) results if fast cluster growth coincides with the transition from subcritical to supercritical conditions. A figure analogous to Fig. 3 for the steplike increase of the maximal cluster size is provided in the supplementary material. Figure 4(a) shows the maximum cluster size observed $\left(n_{\max }\right)$ as a function of $\ln S$ for two water measurements performed with nitrogen and argon as the carrier gas in two different supersaturation regimes of $\ln S \sim 41$ and $\ln S \sim 104$, respectively. $S$ was calculated using the formula and parameters given in Ref. 46 for the liquid-vapor phase transition. The formula and parameters are provided in the supplementary material. As a caveat, we note that the extrapolation to our very low temperatures goes beyond the tested validity range of these expressions. At lower $S(\ln S \sim 41)$, a steplike increase of $n_{\max }$ with increasing $S$ is observed, while at higher $S(\ln S \sim 104)$, $n_{\max }$ increases gradually with increasing $S$. As discussed in more detail in Refs. 12 and 13, the two different types of behavior could be indicative of the presence or absence of a nucleation barrier. In the presence of a nucleation barrier, a steplike increase of $n_{\max }$ is to be expected, while a gradual increase hints at a barrierless growth process (spinodal decomposition).

The experimental mass spectra allow us to specify a range $\left[n_{\max }^{<}, n_{\mathrm{M}}\right]$ for the size of the critical nucleus $n_{\mathrm{c}}$. This range is directly extracted from the last subcritical and the first supercritical mass spectrum. ${ }^{12,13} n_{\max }^{<}$is the largest cluster size in the last subcritical mass spectrum just before nucleation sets in and $n_{\mathrm{M}}$ is the mean of the smallest and largest cluster size observed in the first supercritical spectrum after nucleation sets in. $n_{\mathrm{M}}$ 

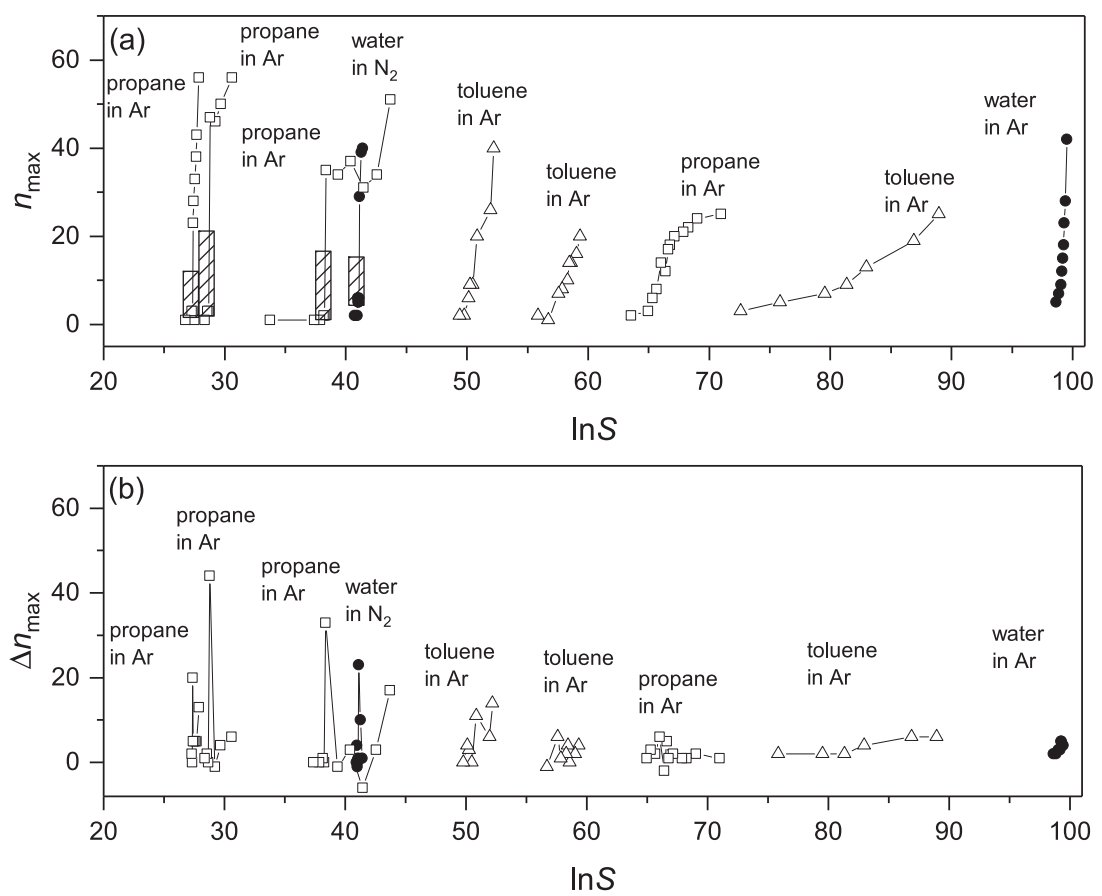

FIG. 4. (a) Maximum cluster size $n_{\max }$ extracted from the cluster mass spectra as a function of $\ln S$. Filled circles: water nucleation experiments using argon and nitrogen as carrier gases. Open squares: propane nucleation experiments using argon as the carrier gas. ${ }^{12}$ Open triangles: toluene nucleation experiments using argon as the carrier gas. ${ }^{13}$ A steplike increase of $n_{\max }$ as a function of $\ln S$ is observed at lower supersaturations which changes to a gradual increase at higher supersaturations. The dashed area indicates the range for the critical cluster size $n_{\mathrm{c}} \in\left[n_{\mathrm{max}}^{<}, n_{\mathrm{M}}\right]$. (b) $\Delta n_{\max }$ as a function of $\ln S$ for the same data as in (a). A steplike behavior is indicated here by a maximum in $\Delta n_{\max }$, while the lack of such a maximum indicates a gradual behavior. presumably overestimates the upper limit for $n_{\mathrm{c}}$ because as soon as nucleation takes place, cluster growth happens spontaneously. For this reason, we believe that $n_{\mathrm{c}}$ probably lies closer to $n_{\max }^{<}$than to $n_{\mathrm{M}}$. The size range of the critical cluster is indicated by the dashed areas in Fig. 4(a). For the water measurements at $\ln S \sim 41$, we determine a critical cluster size range of $\left[n_{\max }^{<}, n_{\mathrm{M}}\right]=[6,15]$ (Table II). For the experimental data at very high supersaturation $(\ln S \sim 104)$, no critical cluster size range can be determined because the gradual increase of $n_{\max }$ hints at a barrierless process, which is consistent with a "critical" cluster size of $n_{\mathrm{c}}=1$.

Figure 4 compares the water nucleation data with our previous data for propane and toluene. ${ }^{12,13}$ For propane, we have also added a new data set at $\ln S \sim 65$. This comparison of the nucleation behavior over a wide range of $S$ values for different types of substances clearly reveals a systematic trend: a steplike behavior is observed at low values of $S$, which at higher values of $S$ turns into a gradual behavior. This is illustrated in Fig. 4(b) by plotting the difference $\Delta n_{\max }$ between the values of $n_{\max }$ at two adjacent data points as a function of $\ln S$. In this graph, a sharp maximum in $\Delta n_{\max }$ indicates a steplike behavior, while the absence of such a maximum indicates a

TABLE II. Comparison of the nucleation rates $J$ as determined in the present work: directly from experimental data, from CNT, ${ }^{49}$ from the Wölk model, ${ }^{2}$ and from MKNT. ${ }^{30,54} n_{\mathrm{c}}$ are the corresponding critical cluster sizes.

\begin{tabular}{lccccc}
\hline \hline & \multicolumn{2}{c}{$J\left(\mathrm{~cm}^{-3} \mathrm{~s}^{-1}\right)$} & & \multicolumn{2}{c}{$n_{\mathrm{c}}$} \\
\cline { 2 - 3 } \cline { 5 - 6 } & $\begin{array}{c}\text { M40 Ar } \\
(\ln S \sim 104)\end{array}$ & $\begin{array}{c}\mathrm{M} 40 \mathrm{~N}_{2} \\
(\ln S \sim 41)\end{array}$ & & $\begin{array}{c}\text { M40 Ar } \\
(\ln S \sim 104)\end{array}$ & $\begin{array}{c}\text { M40 N } 2 \\
(\ln S \sim 41)\end{array}$ \\
\hline Experiment & $2 \cdot 10^{15}$ & $5 \cdot 10^{15}$ & & 1 & {$[6,15]$} \\
CNT & $2 \cdot 10^{13}$ & $1 \cdot 10^{15}$ & & 0.2 & 0.4 \\
Wölk & $5 \cdot 10^{60}$ & $3 \cdot 10^{35}$ & & 0.2 & 0.4 \\
MKNT & $3 \cdot 10^{17}$ & $3 \cdot 10^{18}$ & & 1 & 1 \\
\hline \hline
\end{tabular}

gradual behavior. In principle, this general trend as a function of increasing $\ln S$ is in agreement with the expected dependence of a nucleation barrier on the supersaturation: nucleation kinetics controlled by a barrier at low $S$ is replaced by barrierless growth as the barrier eventually vanishes at high $S$.

\section{B. Nucleation rates from varying the nucleation time}

In the second type of experiment, we varied the nucleation time $t$ systematically with a temporal resolution of $2 \mu \mathrm{s}$ by increasing the axial distance $l$ in the postnozzle flow at constant $S$. This provides cluster size distributions as a function of the nucleation time $t$, from which the cluster number concentrations $N_{\mathrm{n}}^{\mathrm{H}_{2} \mathrm{O}}$ can be retrieved (Sec. II B). $N_{\text {cluster,to }}^{\mathrm{H}_{2} \mathrm{O}}$ is then defined as the sum over all cluster number concentrations $N_{\mathrm{n}}^{\mathrm{H}_{2} \mathrm{O}}$ with $n>1$. Figure 5(a) shows $N_{\text {cluster.tot }}^{\mathrm{H}_{2} \mathrm{O}}$ as a function of $t$ for the two measurement sets recorded at $\ln S=41$ in $\mathrm{N}_{2}$ carrier gas and at $\ln S=104$ in Ar carrier gas. Note that because $N_{\text {cluster,tot }}^{\mathrm{H}_{2} \mathrm{O}}$ is orders of magnitude smaller than the water monomer number concentration $\left(\sim 10^{14} \mathrm{~cm}^{-3}\right)$, monomer depletion and heat release during nucleation are negligible and $S$ stays approximately constant. If summed only over cluster sizes larger than the critical cluster $n>n_{\mathrm{c}}$, the temporal evolution of $N_{\text {cluster,tot }>n_{\mathrm{c}}}^{\mathrm{H}_{2} \mathrm{O}}$ provides direct experimental access to the nucleation rate $J$, at least at early nucleation times when monomer depletion and more complex growth processes, such as cluster coagulation and agglomeration, can be neglected. In this time regime, $N_{\text {cluster,tot }>n_{\mathrm{c}}}^{\mathrm{H}_{2} \mathrm{O}}$ is proportional to $t$, with the nucleation rate

$$
J=\frac{\partial N_{\text {cluster,tot>n}}^{\mathrm{H}_{2} \mathrm{O}}(t)}{\partial t} .
$$

The extraction of $J$ from such linear fits is equivalent to the approaches used for the analysis of results from molecular dynamics simulations. ${ }^{20,22}$ At the lower supersaturation, we have determined $n_{\mathrm{c}}$ between 6 and 15, and at the higher 
supersaturation $n_{\mathrm{c}}=1$ (Sec. III A, Table II). A few examples at $\ln S=41$ together with the corresponding linear fits are shown in Fig. 5(b). The corresponding nucleation rate constants vary in a narrow range between $J=4-5 \cdot 10^{15} \mathrm{~cm}^{-3} \mathrm{~s}^{-1}$ (Table II lists the value for $n_{\mathrm{c}}=6$ ). At $\ln S=104$, a rate of $J=2 \cdot 10^{15}$ $\mathrm{cm}^{-3} \mathrm{~s}^{-1}$ is retrieved from a linear fit for $n_{\mathrm{c}}=1$. The latter rate is rather a growth rate than a nucleation rate because the process is likely barrierless.

Another less direct way to derive rates from the experimental number concentration $N_{\mathrm{n}}^{\mathrm{H}_{2} \mathrm{O}}$ is described in Refs. 29 and 47. It assumes simple gas kinetic collisions and it requires that the steady state condition is reached. The time evolution of the number concentration of water clusters containing $n$ molecules ( $n$-mers) is given by

$$
\frac{\partial N_{\mathrm{n}}^{\mathrm{H}_{2} \mathrm{O}}}{\partial t}=J_{\mathrm{n}-1}-J_{\mathrm{n}}
$$

where $J_{n}$ is the net number of the transitions from $n$-mers to $(n+1)$-mers per unit volume per unit time and is given by
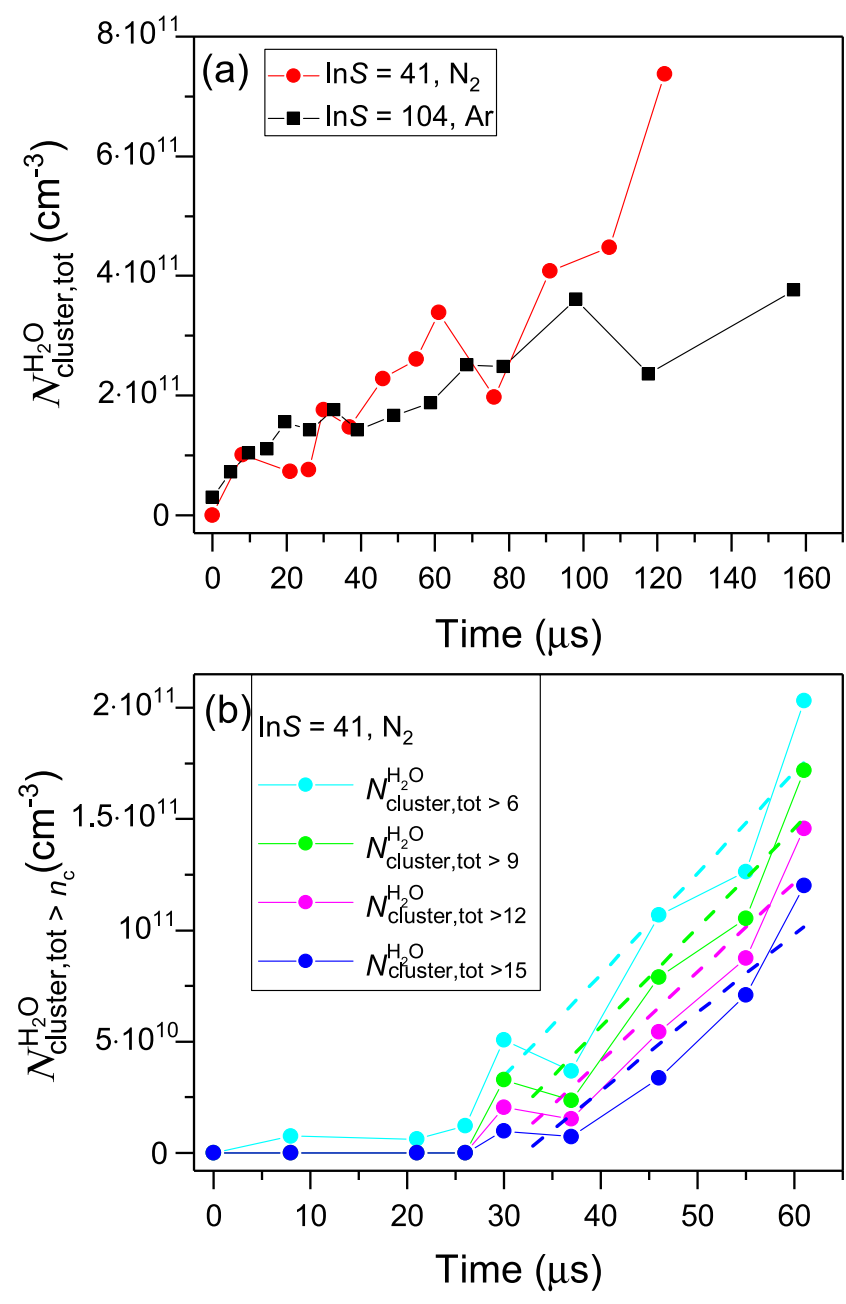

FIG. 5. (a) The total cluster number concentration $N_{\text {cluster,tot }}^{\mathrm{H}_{2} \mathrm{O}}$ as a function of the nucleation time $t$ for water nucleation experiments using nitrogen (circles, $\ln S=41$ ) and argon (squares, $\ln S=104$ ) as the carrier gas. (b) The total number concentration $N_{\text {cluster,tot }>n_{\mathrm{c}}}^{\mathrm{H}_{2} \mathrm{O}}$ for cluster with $n>n_{\mathrm{c}}$ as a function of the nucleation time $t$ for water nucleation experiments using nitrogen (circles, $\ln S=41)$. The differently colored circles correspond to different choices for the value of $n_{\mathrm{c}}$ of $6,9,12$, and 15 . The dashed lines indicate the linear fits [Eq. (2)].

$$
J_{\mathrm{n}}=R^{+}(n) N_{\mathrm{n}}^{\mathrm{H}_{2} \mathrm{O}}-R^{-}(n+1) N_{\mathrm{n}+1}^{\mathrm{H}_{2} \mathrm{O}},
$$

where $R^{+}(n)$ is the rate constant for the transition from $n$-mer to $(n+1)$-mer, i.e., accretion, and $R^{-}(n)$ is the rate constant for the transition from $n$-mer to $(n-1)$-mer, i.e., evaporation of the $n$-mer. $R^{+}(n)$ is given by $R^{+}(n)=\alpha N_{1}^{\mathrm{H}_{2} \mathrm{O}} v_{\text {th }}\left(\pi r_{0}^{2} n^{2 / 3}\right)$, where $\alpha$ is the sticking probability, $v_{t h}$ is the thermal velocity, and $r_{0}$ is the radius of a monomer. We assume $\alpha=1$ for all cluster sizes. From the principle of detailed balance in thermal equilibrium, one finds that the evaporation rate is proportional to $N_{\mathrm{n}, \mathrm{e}}^{\mathrm{H}_{2} \mathrm{O}} / N_{\mathrm{n}+1, \mathrm{e}}^{\mathrm{H}_{2} \mathrm{O}} \propto \exp (-\ln S)$, where $N_{\mathrm{n}, \mathrm{e}}^{\mathrm{H}_{2} \mathrm{O}}$ is the equilibrium number concentration of the $n$-mer. For clusters larger than the critical cluster, the evaporation rate thus becomes very small at $S \gg 1$. For simplicity, we assume here that evaporation is negligible for all cluster sizes, which results in

$$
J_{\mathrm{n}} \simeq R^{+}(n) N_{\mathrm{n}}^{\mathrm{H}_{2} \mathrm{O}} .
$$

In the steady state condition, $J_{\mathrm{n}}$ is constant with respect to $n$ and can be regarded as the nucleation rate, i.e., $J \simeq J_{\mathrm{n}} \cdot{ }^{47} \mathrm{Fig}$ ures 6(a) and 6(b) show $J_{\mathrm{n}}$ as a function of $n$ for different nucleation times $t$ at $\ln S=41$ and $\ln S=104$, respectively. For larger clusters, we find $J_{\mathrm{n}}$ to be approximately constant with respect to $n$, indicating that the system might be almost in steady state.

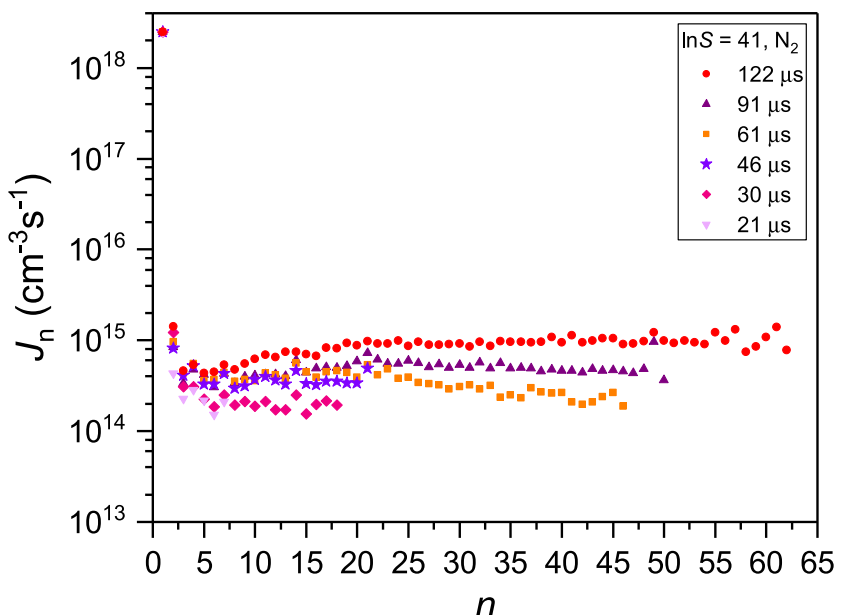

(a)

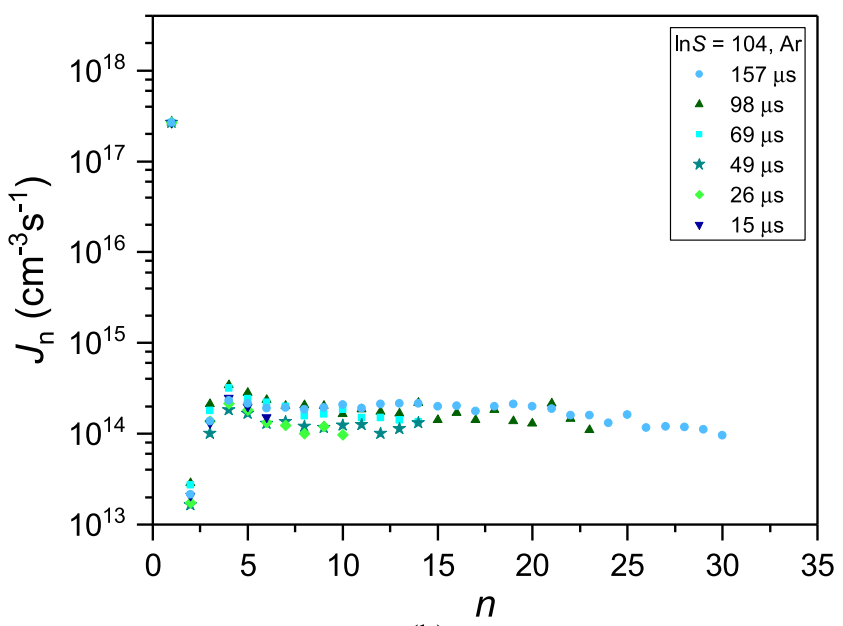

(b)

FIG. 6. The growth rates $J_{\mathrm{n}}$ as a function of $n$ for different nucleation times $t$ as determined from Eq. (4). ${ }^{29}$ (a) For water clusters in $\mathrm{N}_{2}$ carrier gas at $\ln S=41$. (b) For water clusters in $\operatorname{Ar}$ at $\ln S=104$. 
A large deviation is found for $n=1$ even at $\ln S=104$ where $n_{\mathrm{c}}$ is consistently found to be 1 (see also below). The assumption that $\alpha=1$ seems to be particularly bad for the monomer and very small clusters (Sec. IV). Furthermore, despite the high value of $S$, it is not clear how good the assumption of negligible evaporation for the very small clusters is. Assuming that $J \simeq J_{\mathrm{n}}$ and averaging over the constant parts and different times in Fig. 6 results in $J \simeq 6 \cdot 10^{14} \mathrm{~cm}^{-3} \mathrm{~s}^{-1}$ at $\ln S=41$ and $J \simeq 2 \cdot 10^{14} \mathrm{~cm}^{-3} \mathrm{~s}^{-1}$ at $\ln S=104$. While the ratio of the rates at different $S$ is similar, their absolute values are about an order of magnitude smaller than those determined directly from linear fits to $N_{\text {cluster,tot }}^{\mathrm{H}_{2} \mathrm{O}}$ (Fig. 5 and Table II). The expression for $R^{+}(n)$ assumes gas kinetic collisions cross sections that neglect intermolecular interactions. Because of strong long-range electrostatic interactions, one would expect a higher prefactor and thus a higher rate. This could be one of the reasons why the rates derived directly from the time-dependent cluster densities lie higher. Lengyel et al. ${ }^{48}$ showed that the clusters in this size range might not be spherical, which could be another reason why the rates using the above described approach are lower. However, it is difficult to disentangle the individual contributions of the numerous assumptions and simplifications implicit in the second approach.

\section{Nucleation rates and critical clusters from analytical models}

The most attractive comparison of our experimental molecular-level data would be with large scale molecular dynamics (MD) simulations. ${ }^{27}$ Both approaches directly provide the temporal evolution of the cluster size distribution, from which $J$ can be extracted without any further model using the same evaluation method [Eq. (2)] for experiment and theory. For water, however, large scale simulations are currently limited to rates above $10^{19} \mathrm{~cm}^{-3} \mathrm{~s}^{-1}$, which is still above the rates found in the present experiments. Furthermore, the MD simulations are performed at much higher temperatures and lower supersaturations than in this work. These simulations therefore clearly probe a vapor-liquid phase transition. We thus restrict ourselves here to a comparison of our experimental data for $J$ and $n_{\mathrm{c}}$ with corresponding values obtained from three analytical models, which include classical nucleation theory (CNT), an empirical correction model referred to as Wölk model, and mean-field kinetic nucleation theory (MKNT). Input parameters to these models are the experimental values for $T_{\mathrm{F}}$ and $p_{\text {cond }}$, as well as bulk and molecular properties of water.

The most widely used model is classical nucleation theory (CNT). ${ }^{49}$ We calculate $J$ and $n_{\mathrm{c}}$ from

$$
\begin{aligned}
J_{\mathrm{CNT}}= & \sqrt{\frac{2 \cdot \gamma}{\pi \cdot m}} \cdot V_{\mathrm{m}} \cdot\left(\frac{p_{\mathrm{H}_{2} \mathrm{O}}}{k_{\mathrm{B}} \cdot \overline{T_{\mathrm{F}}}}\right)^{2} \\
& \times \exp \left[-\frac{-16 \cdot \pi V_{\mathrm{m}}^{2} \cdot \gamma^{3}}{3 \cdot\left(k_{\mathrm{B}} \cdot \overline{T_{\mathrm{F}}}\right)^{3} \cdot(\ln S)^{2}}\right]
\end{aligned}
$$

and

$$
n_{c}=\left(\frac{2 \cdot \gamma_{\mathrm{red}}}{3 \cdot \ln S}\right)^{3}
$$

where $\gamma$ is the bulk surface tension, $m$ is the molecular mass of the water monomer, $V_{\mathrm{m}}$ is the molecular volume, $p_{\mathrm{H}_{2} \mathrm{O}}$ is the partial pressure of water monomer, $\overline{T_{\mathrm{F}}}$ is the flow temperature, $S$ is the supersaturation, and $\gamma_{\text {red }}$ is the reduced surface tension with $\gamma_{\mathrm{red}}=\frac{\gamma \cdot s_{1}}{k_{\mathrm{B}} \cdot \bar{T}_{\mathrm{F}}}$ with monomer surface area $s_{1}$. The resulting rates and critical nuclei are $J=1 \cdot 10^{15} \mathrm{~cm}^{-3} \mathrm{~s}^{-1}$ and $n_{\mathrm{c}}=0.4$ at $\ln S=41$, and $J=2 \cdot 10^{13} \mathrm{~cm}^{-3} \mathrm{~s}^{-1}$ and $n_{\mathrm{c}}=0.2$ at $\ln S=104$ (Table II). Physically, one would interpret this result as a critical cluster size of 1, i.e., no free energy barrier. As pointed out in Sec. III A, the low temperature is an issue because the values of the equilibrium vapor pressure, the density, and the surface tension are not known at these low temperatures and need to be extrapolated. For lack of better alternatives, we used the formulae and parameters suggested in Ref. 46 for the gas-liquid transition for the extrapolations, with the caveat that our temperatures lie far below the tested range of validity (critical temperature to triple point temperature). The used formulae can be found in the supplementary material. A proper estimate of the associated uncertainties is difficult. However, we can at least provide some information on the sensitivity of $J$ to the variation of the different parameters. A change of the equilibrium vapor pressure of a factor of hundred, for example, changes $J$ by up to an order of magnitude, and if instead of Ref. 46 the extrapolations of Ref. 50 are used, $J$ increases by up to two orders of magnitude. We thus assume uncertainties due to the input parameters to amount to 1-2 orders of magnitude. Given the experimental (Sec. II B) and theoretical uncertainties, the CNT rates seem to be in reasonable agreement with the experimental rates of $J=5 \cdot 10^{15} \mathrm{~cm}^{-3} \mathrm{~s}^{-1}$ at $\ln S=41\left(p_{\mathrm{eq}}=4 \times 10^{-19} \mathrm{~Pa}\right)$ and $J=2 \cdot 10^{15} \mathrm{~cm}^{-3} \mathrm{~s}^{-1}$ at $\ln S=104\left(p_{\mathrm{eq}}=5 \times 10^{-47} \mathrm{~Pa}\right)$. However, it remains unclear how meaningful this comparison is because of the fundamental issues the capillarity approximation in CNT poses at high $S$ where $n_{\mathrm{c}}$ becomes small. As has been shown, $, 151,52$ CNT is not a suitable generalized theory to describe homogeneous nucleation. In our case, there is the additional issue of a value for $n_{\mathrm{c}}<1$ [Eq. (7)]. This is an artifact of the approximate formulation of CNT implicit in Eq. (6), i.e., the replacement of a discrete sum analogous to Eq. (9) by a continuous integral over $n .{ }^{30}$ Without this approximation, the artifact would be removed and CNT would yield the same result as MKNT for our extreme conditions (see below), i.e., $n_{\mathrm{c}}=1$, with the corresponding gas-kinetic collision rate limit of $J=3 \cdot 10^{18} \mathrm{~cm}^{-3} \mathrm{~s}^{-1}$ at $\ln S=41$ and $J=3 \cdot 10^{17} \mathrm{~cm}^{-3} \mathrm{~s}^{-1}$ at $\ln S=104$.

CNT was found to predict a stronger temperature dependence than experimentally observed. ${ }^{50}$ To account for this, it was suggested to employ a two-parameter temperaturedependent correction factor ${ }^{2,50,53}$

$$
J_{\mathrm{Wölk}}=J_{\mathrm{CNT}} \cdot \exp \left(A+\frac{B}{\overline{T_{\mathrm{F}}}}\right),
$$

with $A=-27.56$ and $B=6500 \mathrm{~K}$ for water. The original work expected this parameters to be useful for nucleation rates from $J=10^{0}-10^{20} \mathrm{~cm}^{-3} \mathrm{~s}^{-1}$ for a supersaturation range of $5<S<200$ and a temperature range of $200 \mathrm{~K}<T$ $<310 \mathrm{~K}^{2}$ Later papers showed its validity also beyond this range. ${ }^{1}$ At our experimental conditions, Eq. (8) yields rates of 
$J=3 \cdot 10^{35} \mathrm{~cm}^{-3} \mathrm{~s}^{-1}$ at $\ln S=41$ and $J=5 \cdot 10^{60} \mathrm{~cm}^{-3} \mathrm{~s}^{-1}$ at $\ln S$ $=104$, with the same critical cluster sizes $n_{\mathrm{c}}$ as for CNT. At our low experimental temperatures, the empirical correction apparently yields unreasonable temperature dependencies and rates, which might not be surprising given that our temperatures lie far below the range for which this approach has been validated. Recently, Angélil et al. ${ }^{24}$ have suggested a modified parameter set $A=-20.5$ and $B=6100 \mathrm{~K}$ for Eq. (8), resulting in similarly unreasonable rates.

MKNT includes microscopic effects for small clusters and provides a vanishing free energy barrier at high $S$ values (spinodal decomposition). ${ }^{30,54}$ The theory is based on two parts: a nucleation kinetics part which follows Ref. 55 and a statistical thermodynamics part with mean-field argument. ${ }^{30,54}$ The nucleation rate is given by

$$
J_{\mathrm{MKNT}}=A_{\mathrm{kin}}\left[\sum_{n=1}^{\infty} \exp ^{-H(n)}\right]^{-1},
$$

where $-H(n)=\beta \Delta G(n)=-n \ln S+\theta_{\text {micro }}\left[n^{\mathrm{s}}(n)-1\right]$ for a cluster containing $n$ molecules. $A_{\text {kin }}=\rho_{\mathrm{H}_{2} \mathrm{O}} f_{\text {sat }} S$ is the kinetic prefactor with $f_{\mathrm{sat}}=\frac{p_{\mathrm{eq}}\left(\overline{T_{\mathrm{F}}}\right) s_{1}}{\sqrt{2 \pi m k_{\mathrm{B}} \bar{T}_{\mathrm{F}}}}$ and $s_{1}$ being the surface area of the monomer. The number of surface molecules $n^{\mathrm{s}}(n)$ is calculated from the effective hard sphere diameter $d_{\mathrm{HS}}\left(\overline{T_{\mathrm{F}}}\right){ }^{30}$ We set it at $2.75 \AA$. $\theta_{\text {micro }}=-\ln \left[\frac{\left.-B_{2} p_{\mathrm{eq}} \overline{T_{\mathrm{F}}}\right)}{k_{\mathrm{B}} \overline{T_{\mathrm{F}}}}\right]$ is the microscopic surface tension with the second virial coefficient $B_{2}\left(\overline{T_{\mathrm{F}}}\right)$ from Ref. 56. All other parameters are identical to those used for CNT. ${ }^{46} n_{\mathrm{c}}$ is determined as the position of the minimum of $H(n) .{ }^{30}$ The resulting rates and critical nuclei are $J=3 \cdot 10^{18} \mathrm{~cm}^{-3} \mathrm{~s}^{-1}$ and $n_{\mathrm{c}}=1$ at $\ln S=41$ and $J=3 \cdot 10^{17}$ $\mathrm{cm}^{-3} \mathrm{~s}^{-1}$ and $n_{\mathrm{c}}=1$ at $\ln S=104$ (Table II). In the limit of very high $S$ as in the present study, the MKNT rate equals the gas kinetic collision rate between monomers, i.e., the dimerization rate in Fig. 6 (Sec. III B). As a consequence, the MKNT result under these conditions is insensitive to the input parameters. There is only a small dependence on the liquid density because the molecular size is derived from it. The MKNT rates exceed our direct experimental values by 2-3 orders of magnitude, which is plausible because the gas kinetic collision rate limit is expected to overestimate the dimerization rate (Sec. IV).

\section{DISCUSSION}

Figure 7 compares our rates with data from previous experimental studies, ${ }^{5,6,50,53,57,58}$ from MD simulations, ${ }^{22,24}$ and from a semi-phenomenological (SP) model. ${ }^{24}$ The nucleation rates are shown as a function of $\frac{\ln (S)}{\left(\frac{T_{\mathrm{c}}}{\overline{T_{\mathrm{F}}}}-1\right)^{1.7}}$, which apart from the exponent 1.7 for water is equal to the scaling relation suggested by Hale with an exponent of 1.5. ${ }^{59,60}$ As discussed in Ref. 24, the previous data follow the empirical scaling relation surprisingly well over a wide range of nucleation rates from $J=10^{-2}$ to $J=10^{28} \mathrm{~cm}^{-3} \mathrm{~s}^{-1}$.

The different previous studies cover a temperature range from $\sim 200$ to $400 \mathrm{~K}$ at supersaturations below 150 , while our study was performed at much lower temperatures below $\sim 90 \mathrm{~K}$ and at extremely high supersaturations of more than $10^{17}$. The

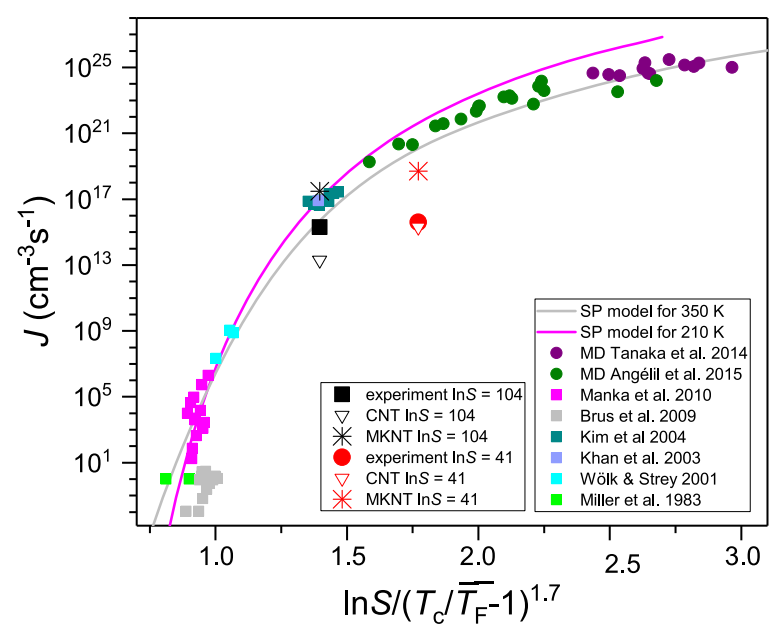

FIG. 7. $J_{\mathrm{n}}$ as a function of $\ln S /\left(T_{c} / \overline{T_{\mathrm{F}}}-1\right)^{1.7}$ from this work (direct experimental data, CNT, and MKNT), an SP model of Ref. 24 for two different temperatures (both using the experimental thermodynamic properties of real water unlike Ref. 24), molecular dynamics simulations using the thermodynamic properties of the SPC/E model (filled circles), ${ }^{22,24}$ and various experimental studies (filled squares). ${ }^{5,6,50,53,58,68} T_{c}=647.15 \mathrm{~K}$ is the critical temperature of water.

previous studies report typical critical cluster sizes in the range $n_{\mathrm{c}}=4-45$, while our study finds $n_{\mathrm{c}}=1$ except for the direct experimental value in $\mathrm{N}_{2}$ carrier gas (Table II). Nevertheless, the rates determined in the present work in $\mathrm{Ar}$ carrier gas $\left(J \sim 10^{15}-10^{17} \mathrm{~cm}^{-3} \mathrm{~s}^{-1}\right.$ at $\left.\ln S=104, \overline{T_{\mathrm{F}}}=47.5 \mathrm{~K}\right)$ to the Hale scaling law curve, i.e., as a function of $\frac{\ln (S)}{\left(\frac{T_{\mathrm{c}}}{\bar{T}_{\mathrm{F}}}-1\right)^{1.7}}$ suggested by previous data. Our rates for $\mathrm{N}_{2}$ carrier gas $\left(J \sim 10^{15}-10^{18} \mathrm{~cm}^{-3} \mathrm{~s}^{-1}\right.$ at $\ln S=41$, $\overline{T_{\mathrm{F}}}=87 \mathrm{~K}$ ), by contrast, lie further off that curve, which would have yielded values of $J \sim 10^{20}-10^{22} \mathrm{~cm}^{-3} \mathrm{~s}^{-1}$. This holds, in particular, for our direct experimental value $J=5 \cdot 10^{15} \mathrm{~cm}^{-3}$ $\mathrm{s}^{-1}$ which lies more than 5 orders of magnitudes below the Hale scaling law behavior, i.e., outside the range of expected uncertainties. In addition, the Hale scaling law predicts a nucleation rate for the $\mathrm{N}_{2}$ measurement, which is much higher than the gas kinetic collision rate (see MKNT). Up to uncertainties in the effective collision cross section, the gas kinetic collision rate is the upper limit for the rate of dimerization and thus also the upper limit for the nucleation rate. As the predictions derived from the Hale scaling law exceed this limit by far, they must appear unreasonable. At our conditions, however, the Hale scaling law behavior is not expected to hold. The Hale scaling law relies on the description by CNT combined with simple assumptions for the temperature dependence of liquid density (constant), vapor pressure (Clausius-Clapeyron), and surface tension (linear). ${ }^{59}$ Consequently, Hale indicated an expected validity in the range $0.3<T / T_{\mathrm{c}}<0.5$, i.e., for water in the range of $T=194-323 \mathrm{~K}$. Our temperatures lie far below that range. Furthermore, the scaling law qualitatively reflects the hyperbolic dependence of the CNT free energy barrier on $\frac{\ln (S)}{\left(\frac{T_{\mathrm{c}}}{T_{\mathrm{F}}}-1\right)^{1.5}}$ resulting from the assumed temperature dependencies. Thus, the apparent agreement of our Ar results with the Hale scaling law must be considered fortuitous, and the deviation we find for our $\mathrm{N}_{2}$ rate is not surprising. 
A direct comparison with previous experimental data is not easily feasible at this stage. The rates determined in Refs. 5 and 6 and Fig. 7 are similar to our rates recorded in $\mathrm{Ar}$ carrier gas, but a direct comparison is not possible because these data were recorded at much higher temperatures (208$237 \mathrm{~K})$ and much lower supersaturations (36-143). To the best of our knowledge, Ref. 9 is the only study that reports cluster growth data at temperatures and supersaturations similar to our study. However, it remains unclear how strongly the experimental data of Ref. 9 were affected by the cluster size dependence of the ionization and detection efficiency and by cluster fragmentation after electron ionization. The latter has been reported to be a major issue even for low electron energies. ${ }^{18,19,61-63}$ Furthermore, the authors of Ref. 9 observe substantial monomer depletion while we do not, and our detection limit lies probably far below theirs because we apply high extraction voltages (Sec. II A). Given these experimental differences and uncertainties, we refrain from a direct comparison with the experimental data.

We can, however, compare with the theoretical model of Ref. 9. As shown in Sec. III C, CNT and MKNT both yield the gas kinetic collision limit for the nucleation rates, which exceeds our direct experimental values by 2-3 orders of magnitude (Table II). The main reason for this discrepancy is presumably the assumption of a unit sticking probability in those models. At the exceedingly low pressure of our experiment, by contrast, one would expect a sticking probability far less than unity for very small clusters, in particular, for the dimerization. Klippenstein and co-workers have presented a master equation model based on $a b$ initio transition state theory. ${ }^{9}$ As such, it explicitly accounts for the pressure and temperature-dependent sticking coefficients. Similar to CNT and MKNT, they also find that the nucleation rate is determined by the dimerization step, but that at pressures between 10 and $100 \mathrm{~Pa}$, the rates lie orders of magnitude below the high pressure limit, which corresponds to a very low sticking probability. From the rate constants quoted in Table S3 of Ref. 9, we interpolate model predictions for the nucleation rates under our experimental conditions of $J=8 \cdot 10^{15} \mathrm{~cm}^{-3} \mathrm{~s}^{-1}$ for $\mathrm{N}_{2}$ and $J=6 \cdot 10^{15} \mathrm{~cm}^{-3} \mathrm{~s}^{-1}$ for Ar, which agree surprisingly well with our direct experimental rates (Table II). For Ar, this is also fully consistent with our findings of a barrierless nucleation, i.e., $n_{\mathrm{c}}=1$. For $\mathrm{N}_{2}$, the picture is less clear cut. While the direct experimental value for the nucleation rate agrees very well with the model of Klippenstein and co-workers, the critical cluster size of $n_{\mathrm{c}}=[6,15]$ that we determine from the steplike behavior in Fig. 4 does not seem to be consistent with this model-at first sight. The steplike behavior that we observe implies a kinetic bottleneck in the vicinity of $n_{\mathrm{c}}$. The standard interpretation of the formation of $n_{\mathrm{c}}$-clusters as the rate determining step of the nucleation kinetics is indeed inconsistent with the $a b$ initio model of Klippenstein, which finds dimerization as the rate determining step for $\mathrm{N}_{2}$ also. As it seems implausible that the $a b$ initio theory is off by several orders of magnitude for the relative growth rates for different clusters, the standard interpretation does not seem to be correct here. In principle, the nature of the carrier gas ( $\mathrm{He}$ vs. $\mathrm{N}_{2}$ ) could play a role (see, for example, Refs. 26, 64, and 65), but we find drastic effects due to the nature of the carrier gas rather unlikely. At this point, we can only speculate about another scenario: still assuming that the primary bottleneck of the kinetics (i.e., the rate determining step) is the dimerization, a secondary bottleneck in the growth kinetics might result from a local maximum in the free energy of cluster formation and/or from a local minimum of the sticking coefficient for the monomer addition. There are interesting analogies between this picture and Wilemski's discussion of the central role of dimerization kinetics in classical theories of nucleation. ${ }^{66}$ Interestingly, Klippenstein and coworkers found such a secondary bottleneck in the form of a sudden decrease of the rate coefficient for monomer addition at $p_{\mathrm{F}}=10-100 \mathrm{~Pa}$ and $\overline{T_{\mathrm{F}}}=100 \mathrm{~K}$ by two orders of magnitude when going from $n=4$ to $n=5$. Only explicit model calculations of the full kinetics could clarify whether this could indeed lead to the steplike behavior we observe in the experiment for $\mathrm{N}_{2}$. It is also noteworthy that Klippenstein and co-workers did not find such a sudden increase of the rate coefficient for larger cluster sizes at $p_{\mathrm{F}}=10-100 \mathrm{~Pa}$ and $\overline{T_{\mathrm{F}}}=50 \mathrm{~K}$, i.e., close to the conditions of our Ar measurements, where we find a gradual behavior instead of a steplike behavior as for $\mathrm{N}_{2}$.

\section{SUMMARY}

Our experimental nucleation studies for water under extreme conditions of low temperatures and very high supersaturations suggest a more complicated behavior than expected at first sight. We observe two different nucleation onset behaviors: a steplike increase of the maximum cluster size with increasing supersaturation versus a gradual increase of the maximum cluster size with increasing supersaturation. We find the gradual increase and also the value of the corresponding experimental nucleation rate to be fully consistent with the expected barrierless growth (critical cluster size of 1) at such high supersaturations. The interpretation of the steplike behavior with an experimentally determined critical cluster size in the region of 6-15, however, is less clear cut and will require explicit model calculation of the full kinetics. We speculate that the steplike behavior might be caused by a secondary bottleneck in the growth kinetics at a cluster size around 6, with the primary bottleneck still being the dimerization. This seems consistent with the sudden decrease of the rate coefficient for the monomer addition to the pentamer as calculated by Klippenstein and co-workers in Ref. 9. A comparison with previous data for propane and toluene reveals that the observation of a continuous change from a steplike to a gradual onset behavior with increasing supersaturation at very high absolute supersaturations is not limited to water. The experimental rates, both for the steplike and for the gradual case, agree very well with the prediction from a master equation model based on $a b$ initio transition state theory by Klippenstein and co-workers. Both the experimental rates and the $a b$ initio rates of Klippenstein and co-workers are 2-3 orders of magnitude lower than the values retrieved from the gas kinetic collision model assuming unit sticking probability. This implies that the actual sticking probability of small clusters is far less than unity, which is consistent with the expectation of the growth kinetics deep in the fall-off region for small clusters at the low pressures of our experiments. 


\section{SUPPLEMENTARY MATERIAL}

See supplementary material for a figure analogous to Fig. 3 for a steplike nucleation behavior and formulae and parameters used for the calculation of the vapor pressure, surface tension, and density of liquid water taken from Ref. 46.

\section{ACKNOWLEDGMENTS}

We thank David Stapfer and Markus Steger from our mechanical and electronic shops for their assistance in developing and maintaining the experimental setup. Financial support was provided by the Swiss National Science Foundation (SNF Project No. 200020-172472) and ETH Zürich.

${ }^{1}$ B. E. Wyslouzil and J. Wölk, J. Chem. Phys. 145, 211702 (2016).

${ }^{2}$ J. Wölk, R. Strey, C. H. Heath, and B. E. Wyslouzil, J. Chem. Phys. 117, 4954 (2002).

${ }^{3}$ A. Manka, H. Pathak, S. Tanimura, J. Wölk, R. Strey, and B. E. Wyslouzil, Phys. Chem. Chem. Phys. 14, 4505 (2012).

${ }^{4}$ C. A. Moses and G. D. Stein, J. Fluids Eng. 100, 311 (1978).

${ }^{5}$ A. Khan, C. H. Heath, U. M. Dieregsweiler, B. E. Wyslouzil, and R. Strey, J. Chem. Phys. 119, 3138 (2003).

${ }^{6}$ Y. J. Kim, B. E. Wyslouzil, G. Wilemski, J. Wölk, and R. Strey, J. Phys. Chem. A 108, 4365 (2004).

${ }^{7}$ B. E. Wyslouzil, G. Wilemski, R. Strey, S. Seifert, and R. E. Winans, Phys. Chem. Chem. Phys. 9, 5353 (2007).

${ }^{8}$ H. Pathak, J. Wölk, R. Strey, and B. E. Wyslouzil, J. Chem. Phys. 140, 034304 (2014).

${ }^{9}$ J. Bourgalais, V. Roussel, M. Capron, A. Benidar, A. W. Jasper, S. J. Klippenstein, L. Biennier, and S. D. Le Picard, Phys. Rev. Lett. 116, 113401 (2016).

${ }^{10}$ B. Schläppi, J. H. Litman, J. J. Ferreiro, D. Stapfer, and R. Signorell, Phys. Chem. Chem. Phys. 17, 25761 (2015).

${ }^{11}$ J. J. Ferreiro, T. E. Gartmann, B. Schläppi, and R. Signorell, Z. Phys. Chem. 229, 1765 (2015).

${ }^{12}$ J. J. Ferreiro, S. Chakrabarty, B. Schläppi, and R. Signorell, J. Chem. Phys. 145, 211907 (2016).

${ }^{13}$ S. Chakrabarty, J. J. Ferreiro, M. Lippe, and R. Signorell, J. Phys. Chem. A 121, 3991 (2017).

${ }^{14}$ B. L. Yoder, J. H. Litman, P. W. Forysinski, J. L. Corbett, and R. Signorell, J. Phys. Chem. Lett. 2, 2623 (2011).

${ }^{15}$ J. H. Litman, B. L. Yoder, B. Schläppi, and R. Signorell, Phys. Chem. Chem. Phys. 15, 940 (2013).

${ }^{16}$ F. Dong, S. Heinbuch, J. J. Rocca, and E. R. Bernstein, J. Chem. Phys. 124, 224319 (2006).

${ }^{17}$ L. Belau, K. R. Wilson, S. R. Leone, and M. Ahmed, J. Phys. Chem. A 111, 10075 (2007).

${ }^{18}$ J. Lengyel, A. Pysanenko, J. Kočišek, V. Poterya, C. C. Pradzynski, T. Zeuch, P. Slavíček, and M. Fárník, J. Phys. Chem. Lett. 3, 3096 (2012).

${ }^{19}$ J. Lengyel, A. Pysanenko, V. Poterya, J. Kočišek, and M. Fárník, Chem. Phys. Lett. 612, 256 (2014).

${ }^{20}$ K. Yasuoka and M. Matsumoto, J. Chem. Phys. 109, 8463 (1998).

${ }^{21}$ H. Matsubara, T. Koishi, T. Ebisuzaki, and K. Yasuoka, J. Chem. Phys. 127, 214507 (2007).

${ }^{22}$ K. K. Tanaka, A. Kawano, and H. Tanaka, J. Chem. Phys. 140, 114302 (2014).

${ }^{23}$ M. Duška, T. Němec, J. Hrubý, V. Vinš, and B. Planková, EPJ Web Conf. 92, 02013 (2015).

${ }^{24}$ R. Angélil, J. Diemand, K. K. Tanaka, and H. Tanaka, J. Chem. Phys. 143, 064507 (2015)

${ }^{25}$ F. Zipoli, T. Laino, S. Stolz, E. Martin, C. Winkelmann, and A. Curioni, J. Chem. Phys. 139, 094501 (2013).
${ }^{26}$ M. A. L. J. Fransen, J. Hrubý, D. M. J. Smeulders, and M. E. H. van Dongen, J. Chem. Phys. 142, 164307 (2015).

${ }^{27}$ J. Diemand, R. Angélil, K. K. Tanaka, and H. Tanaka, J. Chem. Phys. 139, 074309 (2013).

${ }^{28}$ R. Angélil, J. Diemand, K. K. Tanaka, and H. Tanaka, J. Chem. Phys. 140, 074303 (2014).

${ }^{29}$ K. K. Tanaka, J. Diemand, R. Angélil, and H. Tanaka, J. Chem. Phys. 140, 194310 (2014).

${ }^{30}$ V. I. Kalikmanov, Nucleation Theory (Springer Netherlands, Heidelberg, 2013).

${ }^{31}$ J. Wedekind, G. Chkonia, J. Wölk, R. Strey, and D. Reguera, J. Chem. Phys. 131, 114506 (2009).

${ }^{32}$ A. M. Savel'ev and A. M. Starik, Phys. Chem. Chem. Phys. 19, 523 (2017).

${ }^{33}$ G. K. Schenter, S. M. Kathmann, and B. C. Garrett, Phys. Rev. Lett. 82, 3484 (1999)

${ }^{34}$ D. Reguera and H. Reiss, Phys. Rev. Lett. 93, 165701 (2004).

${ }^{35}$ D. Reguera and H. Reiss, J. Phys. Chem. B 108, 19831 (2004).

${ }^{36}$ L. Inci and R. K. Bowles, J. Chem. Phys. 139, 214703 (2013).

${ }^{37}$ S. M. Kathmann, G. K. Schenter, B. C. Garrett, B. Chen, and J. I. Siepmann, J. Phys. Chem. C 113, 10354 (2009).

${ }^{38}$ D. B. Atkinson and M. A. Smith, Rev. Sci. Instrum. 66, 4434 (1995).

${ }^{39}$ P. W. Forysinski, P. Zielke, D. Luckhaus, and R. Signorell, Phys. Chem. Chem. Phys. 12, 3121 (2010).

${ }^{40}$ F. Dong, S. Heinbuch, J. J. Rocca, and E. R. Bernstein, J. Chem. Phys. 125, 154317 (2006).

${ }^{41}$ Y. J. Hu, H. B. Fu, and E. R. Bernstein, J. Chem. Phys. 125, 184308 (2006).

${ }^{42}$ S. Wei, W. B. Tzeng, and A. W. Castleman, Jr., J. Phys. Chem. 95, 5080 (1991).

${ }^{43}$ K. Kameta, N. Kouchi, M. Ukai, and Y. Hatano, J. Electron Spectrosc. Relat. Phenom. 123, 225 (2002).

${ }^{44}$ G. N. Haddad and J. A. R. Samson, J. Chem. Phys. 84, 6623 (1986).

${ }^{45}$ R. B. Opsal, K. G. Owens, and J. P. Reilly, Anal. Chem. 57, 1884 (1985).

${ }^{46}$ V. Deutscher Ingenieure, G. Verfahrenstechnik, and Chemieingenieurwesen, VDI-Wärmeatlas (Springer Vieweg, 2013).

${ }^{47}$ J. Feder, K. C. Russell, J. Lothe, and G. M. Pound, Adv. Phys. 15, 111 (1966).

${ }^{48}$ J. Lengyel, A. Pysanenko, V. Poterya, P. Slavíček, M. Fárník, J. Kočišek, and J. Fedor, Phys. Rev. Lett. 112, 113401 (2014).

${ }^{49}$ R. Becker and W. Döring, Ann. Phys. 416, 719 (1935).

${ }^{50}$ J. Wölk and R. Strey, J. Phys. Chem. B 105, 11683 (2001).

${ }^{51}$ S. Sinha, A. Bhabhe, H. Laksmono, J. Wölk, R. Strey, and B. Wyslouzil, J. Chem. Phys. 132, 064304 (2010).

${ }^{52}$ K. Iland, J. Wölk, R. Strey, and D. Kashchiev, J. Chem. Phys. 127, 154506 (2007).

${ }^{53}$ A. A. Manka, D. Brus, A.-P. Hyvärinen, H. Lihavainen, J. Wölk, and R. Strey, J. Chem. Phys. 132, 244505 (2010).

${ }^{54}$ V. I. Kalikmanov, J. Chem. Phys. 124, 124505 (2006).

${ }^{55}$ J. L. Katz and H. Wiedersich, J. Colloid Interface Sci. 61, 351 (1977).

${ }^{56}$ A. Dillmann and G. E. A. Meier, J. Chem. Phys. 94, 3872 (1991).

${ }^{57}$ D. Brus, A.-P. Hyvärinen, V. Ždímal, and H. Lihavainen, J. Chem. Phys. 122, 214506 (2005).

${ }^{58}$ R. C. Miller, R. J. Anderson, J. L. Kassner, and D. E. Hagen, J. Chem. Phys. 78, 3204 (1983).

${ }^{59}$ B. N. Hale, Phys. Rev. A 33, 4156 (1986).

${ }^{60}$ B. N. Hale, J. Chem. Phys. 122, 204509 (2005).

${ }^{61}$ U. Buck, R. Krohne, and S. Schütte, J. Chem. Phys. 106, 109 (1997).

${ }^{62}$ U. Buck and C. Steinbach, J. Phys. Chem. A 102, 7333 (1998).

${ }^{63}$ S. Schütte and U. Buck, Int. J. Mass Spectrom. 220, 183 (2002).

${ }^{64}$ L. R. Dumitrescu, H. Huinink, D. M. J. Smeulders, J. A. M. Dam, and S. V. Gaastra-Nedea, J. Chem. Phys. 148, 194502 (2018).

${ }^{65} \mathrm{~J}$. Wedekind, A.-P. Hyvärinen, D. Brus, and D. Reguera, Phys. Rev. Lett. 101, 125703 (2008).

${ }^{66}$ G. Wilemski, J. Chem. Phys. 103, 1119 (1995).

${ }^{67}$ B. Schläppi, J. J. Ferreiro, J. H. Litman, and R. Signorell, Int. J. Mass Spectrom. 372, 13 (2014).

${ }^{68}$ D. Brus, V. Ždímal, and J. Smolík, J. Chem. Phys. 129, 174501 (2008). 